\title{
Efficient Synthesis of 2-(Trimethylsilyl)phenyl Trifluoromethanesulfonate: A Versatile Precursor to ortho-Benzyne
}

\author{
Sarah M. Bronner and Neil K. Garg* \\ Department of Chemistry and Biochemistry, University of California \\ Los Angeles, California 90095 \\ $\underline{\text { Supporting Information - Table of Contents }}$
}

Materials and Methods.

${ }^{1} \mathrm{H}$ NMR and ${ }^{13} \mathrm{C}$ NMR Spectra. S2-S8

Materials and Methods. Unless stated otherwise, reactions were conducted in flame-dried glassware under an atmosphere of nitrogen using anhydrous solvents (either freshly distilled or passed through activated alumina columns). All commercially available reagents were used as received, with the exception of TMSCl (distilled over $\mathrm{CaH}_{2}$ ) and TBSOTf (distilled). $\mathrm{N}$ phenylbis(trifluoromethanesulfonimide) was obtained from Oakwood Products, Inc. Thin-layer chromatography (TLC) was conducted with EMD gel 60 F254 pre-coated plates $(0.25 \mathrm{~mm})$ and visualized using a combination of UV, anisaldehyde, iodine, and potassium permanganate staining. EMD silica gel 60 (particle size $0.040-0.063 \mathrm{~mm}$ ) was used for flash column chromatography. ${ }^{1} \mathrm{H}$ NMR spectra (at $300 \mathrm{MHz}, 400 \mathrm{MHz}$, or $500 \mathrm{MHz}$ ) are reported relative to deuterated solvent signals. Data for ${ }^{1} \mathrm{H}$ NMR spectra are reported as follows: chemical shift $(\delta$ ppm), multiplicity, coupling constant $(\mathrm{Hz})$ and integration. ${ }^{13} \mathrm{C}$ NMR spectra are reported in terms of chemical shift. IR data are reported in terms of frequency absorption (cm-1). 


\section{${ }^{1} \mathrm{H}$ NMR and ${ }^{13} \mathrm{C}$ NMR Spectra for Compounds 15, 17, 2:}




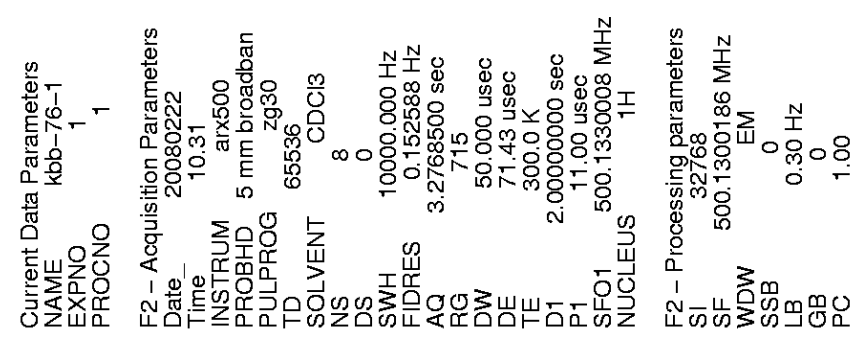

$66 \mathcal{G}^{\prime} \textrm{ }$

$908 \cdot 1$
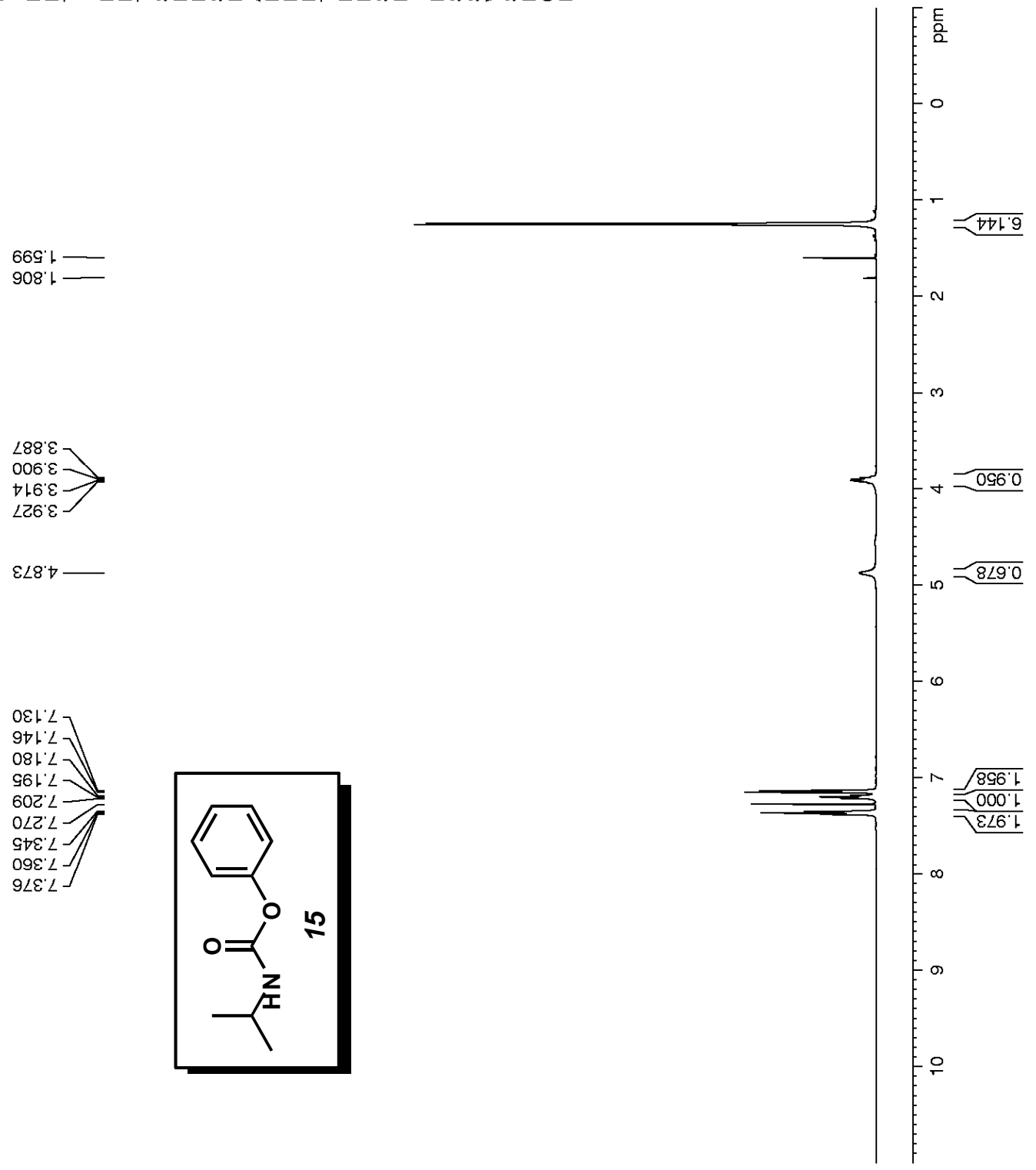


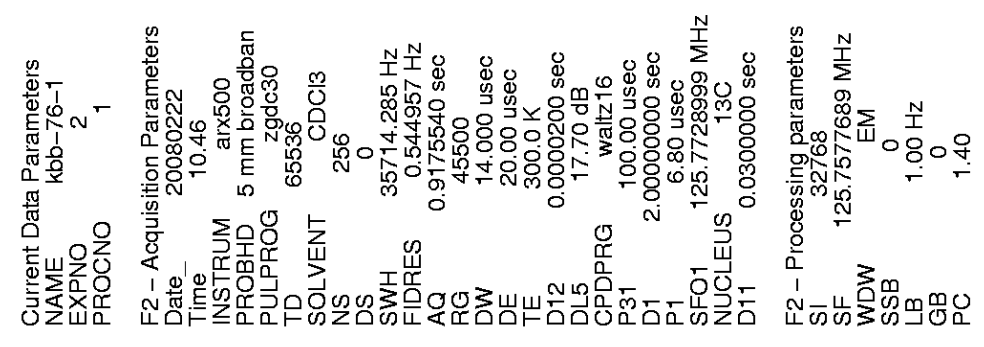

$960 \cdot \varepsilon z-$

†เ9' $\varepsilon \triangleright$

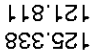

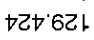

E9Z'เG

G98' $\varepsilon \mathrm{GL}$

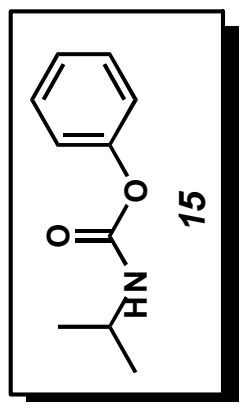

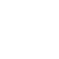



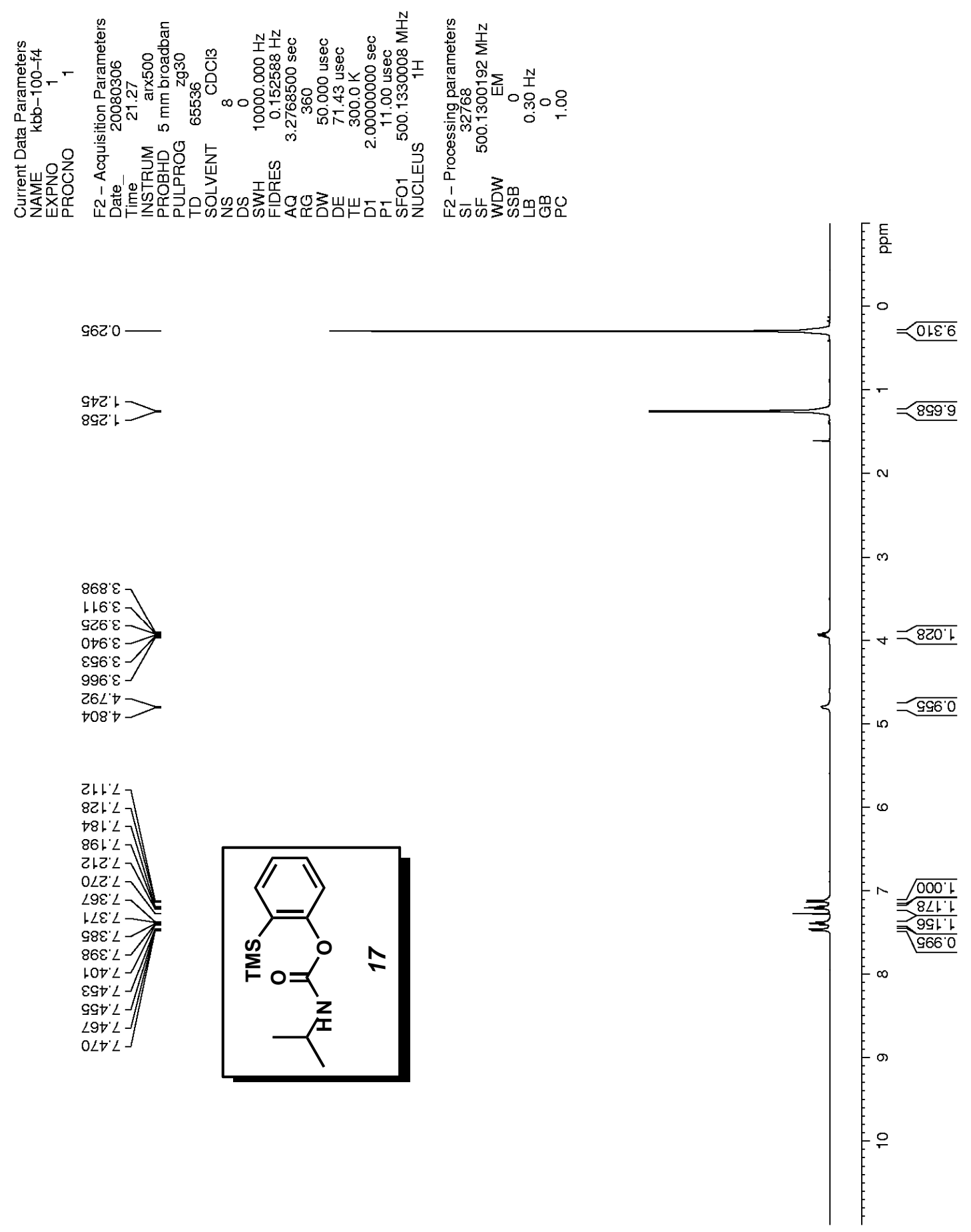

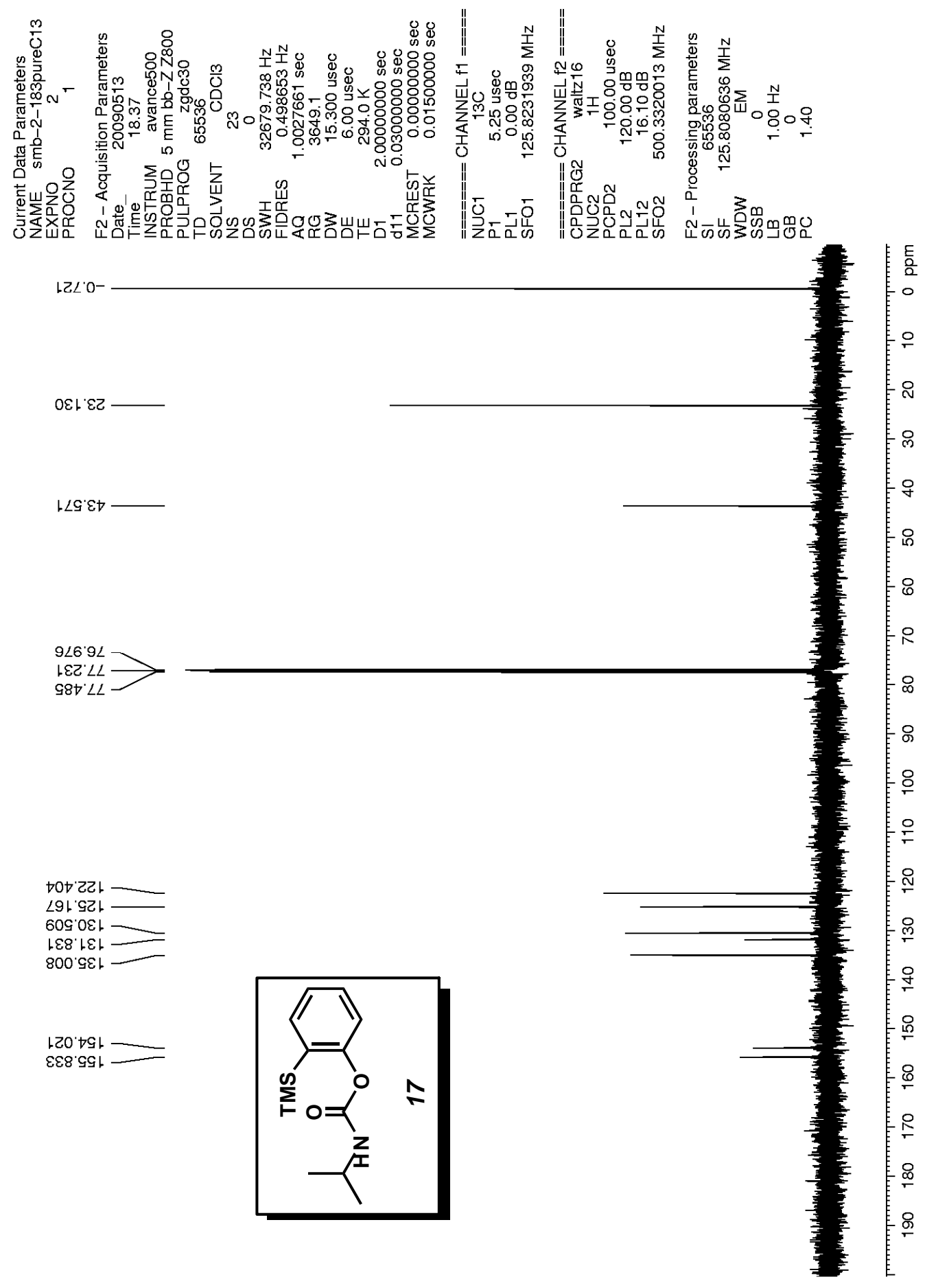

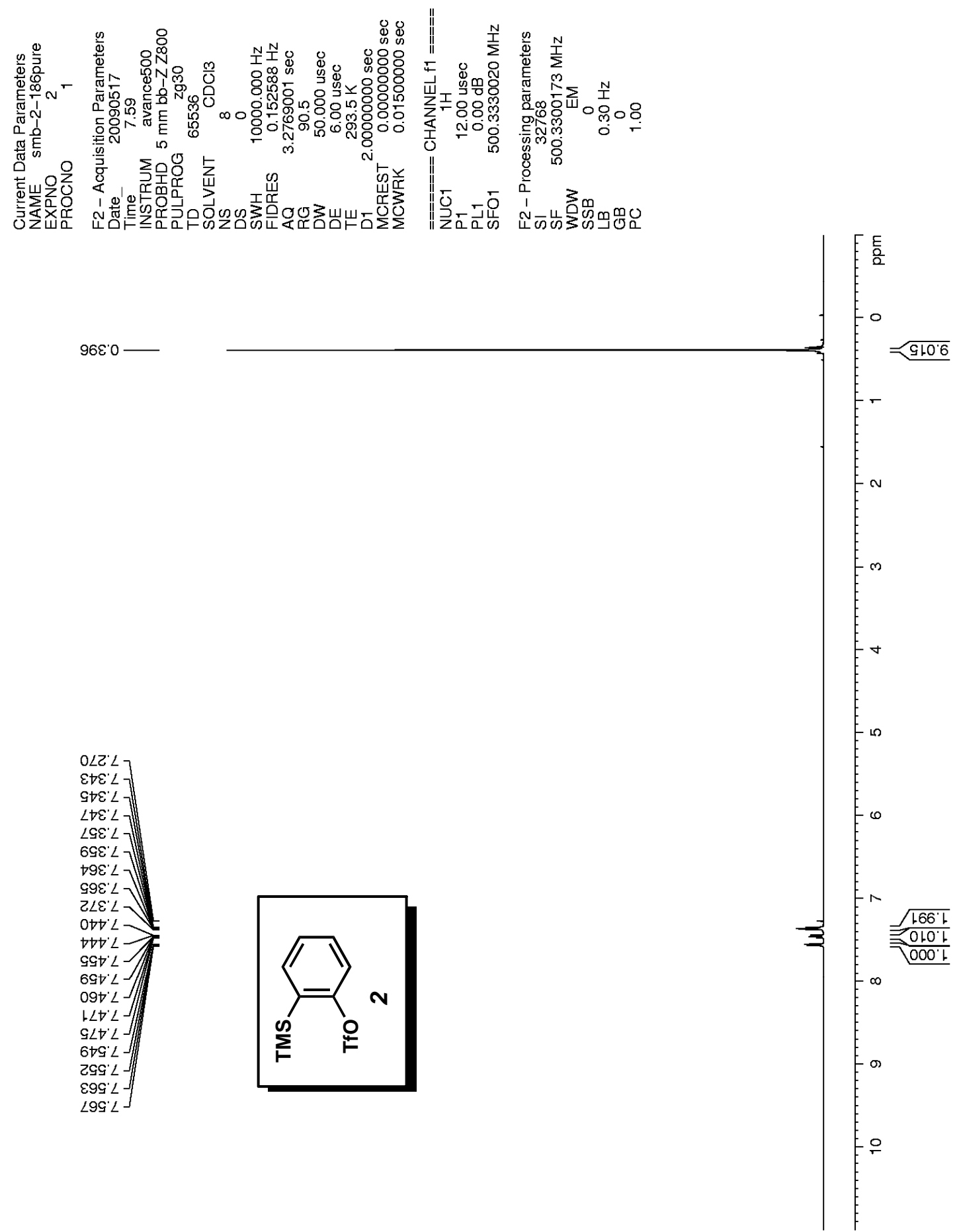

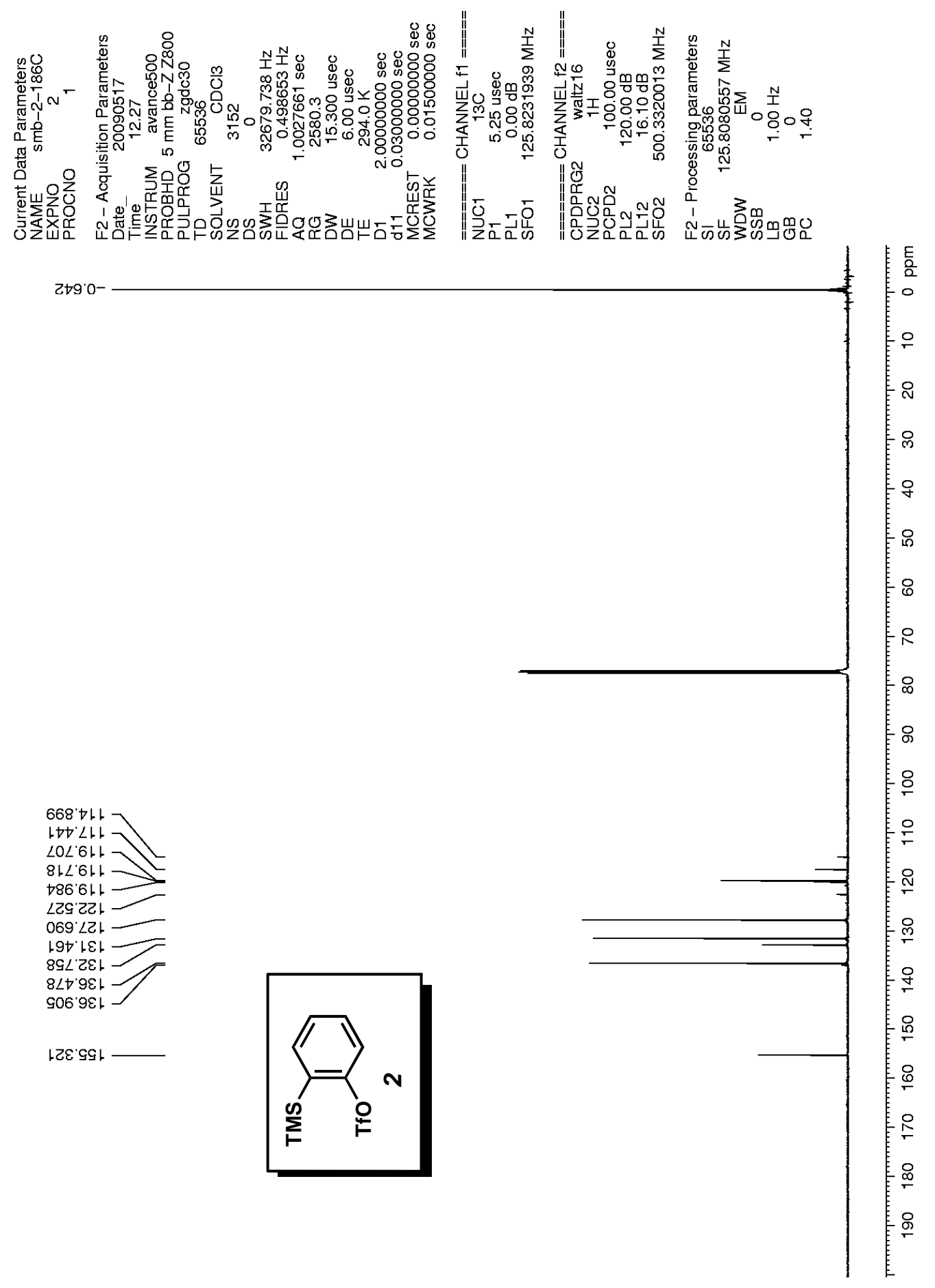